Cahiers $d u$ MONDE RUSSE

\section{Cahiers du monde russe}

Russie - Empire russe - Union soviétique et États indépendants

41/2-3 2000

En islam sibérien

\title{
Le syncrétisme islam-paganisme chez les peuples türks de Sibérie occidentale
}

\author{
Aleksandr G. Seleznev
}

\section{OpenEdition}

Journals

Édition électronique

URL : https://journals.openedition.org/monderusse/50

DOI : 10.4000/monderusse. 50

ISSN : $1777-5388$

Éditeur

Éditions de l'EHESS

Édition imprimée

Date de publication : 1 avril 2000

Pagination : 341-356

ISBN : 2-7132-1361-4

ISSN : $1252-6576$

Référence électronique

Aleksandr G. Seleznev, «Le syncrétisme islam-paganisme chez les peuples türks de Sibérie occidentale », Cahiers du monde russe [En ligne], 41/2-3 | 2000, mis en ligne le 15 janvier 2007, consulté le 02 septembre 2022. URL : http://journals.openedition.org/monderusse/50 ; DOI : https:// doi.org/10.4000/monderusse. 50 


\section{LE SYNCRÉTISME ISLAM-PAGANISME CHEZ LES PEUPLES TÜRKS DE SIBÉRIE OCCIDENTALE}

SELON UNE LÉGENDE TOUJOURS VIVACE dans la mémoire populaire, c'est en 13941395 que les premiers propagateurs de l'islam auraient fait leur apparition en Sibérie occidentale. Cette légende a fini par être prise pour fait historique dans la société russe actuelle, en proie à un réveil brutal et impétueux de ses identités ethniques. Au point que les villes d'Omsk et de Tioumen, situées au centre de districts dotés de populations musulmanes compactes de Tatars et de Qazaqs, ont fêté récemment le 600 anniversaire de l'islam sibérien - célébration à la fois religieuse et nationale, avec festivals de culture traditionnelle et conférences académiques.

La population musulmane de Sibérie est constituée de trois grands groupes ethniques : les Tatars de Sibérie, les Tatars de la Volga et de l'Oural, les Qazaqs de Sibérie occidentale. Les premiers se subdivisent eux-mêmes en trois composantes ethno-territoriales : les Tatars de Tomsk, ceux du Tobol et de l'Irtysh et, enfin, les Tatars de Baraba. Les populations migrantes venue de la Volga et de l'Oural (Tatars de Kazan, Michars, Tatars christianisés - krjašeny - , etc.) se sont installées en Sibérie entre le $\mathrm{XVI}^{\mathrm{e}}$ et le $\mathrm{XX}^{\mathrm{e}}$ siècle. Ces différents groupes tatars, « autochtones » ou « migrants », se répartissent, au milieu de Russes et d'autres populations, entre les régions (oblasti) de Tioumen, Omsk, Novosibirsk, Kemerovo et Tomsk.

Le groupe qazaq sibérien est réparti, lui, dans les districts (rajony) méridionaux des régions de Novosibirsk, Omsk et Tioumen, et le territoire (kraj) de l'Altaï. Les premières populations qazaques sont apparues en Sibérie aux $\mathrm{XV}^{\mathrm{e}}-\mathrm{XVI}^{\mathrm{e}}$ siècles, mais c'est à partir du XVIII ${ }^{\mathrm{e}}$ siècle que la région a véritablement commencé à faire l'objet d'un véritable peuplement qazaq. La Sibérie occidentale a accueilli principalement des Qazaqs de la Horde médiane : Qiptchaq, Arghyn, Naïman, Kerey et Wak (ou Waq). La propagation de l'islam chez les Qazaqs a coïncidé avec la conversion des Tatars de Sibérie.

Il ne fait pas de doute que l'islam sibérien représente en soi un phénomène historique dont la résonnance est à l'échelle de toute l'umma. Il constitue avant tout le 
point le plus avancé de la civilisation islamique vers le nord. En même temps, l'étude de cet islam pose un certain nombre de problèmes spécifiques, sur lesquels nous allons revenir ici. Tout un ensemble de questions est notamment lié à l'influence culturelle de l'islam sur les populations türkophones de la plaine de Sibérie occidentale. Selon certains auteurs, cette influence a donné lieu à la formation, chez les Tatars et les Qazaqs que l'on rencontre en Sibérie, d'un phénomène particulier : une forme de syncrétisme islam-paganisme ${ }^{1}$. Le présent article est consacré à un examen de divers aspects de cette problématique.

Pour commencer, il nous faut d'abord revenir en quelques mots sur cette fameuse date de 1394-1395, supposée être celle du commencement de la propagation de l'islam en Sibérie. L'histoire de l'humanité suggère que la diffusion d'une religion est un processus toujours complexe, conditionné par toute une combinaison de facteurs économiques et sociaux, ethniques, culturels ou politiques. Il n'y a d'ailleurs pas unanimité, chez les historiens russes, sur la chronologie de la propagation de l'islam en terre sibérienne.

Selon le point de vue le plus répandu, l'islam aurait été adopté comme religion officielle du khanat de Sibérie dans les années 1570, sous le khan Kuchum². Mais cette opinion n'est pas la seule. La datation la plus ancienne de la pénétration de l'islam en Sibérie est proposée par un ethnologue du Tatarstan, Foat Väleev, qui affirme : «On peut supposer la possibilité d'une propagation de l'islam parmi les ancêtres des Tatars de Sibérie, directement par les religieux arabes venus au $\mathrm{X}^{\mathrm{e}}$ siècle chez les Bulghars de la Volga avec l'ambassade du caliphe de Bagdad ${ }^{3}$ ». La chronologie la plus tardive du même événement est celle que propose le célèbre orientaliste russe de la première moitié du XXe siècle Vladimir V. Barthold - selon lequel l'islam ne s'est répandu parmi les Türks de Sibérie qu'après l'arrivée des populations russes depuis le nord, en remontant le cours de l'Irtysh ${ }^{4}$. Cette opinion est confirmée par les découvertes archéologiques, qui attestent l'absence quasigénérale des attributs distinctifs du culte islamique sur les sites de Sibérie occidentale, aux XVII et $\mathrm{XVIII}^{\mathrm{e}}$ siècles ${ }^{5}$ : il est manifeste qu'à cette époque l'islam restait encore étranger aux couches les plus larges de la population.

1. I. V. Belič, « Priroda v doislamskih verovanijah sibirskih tatar » (La nature dans les croyances préislamiques des Tatars de Sibérie), in Kulturnye $i$ hozjajstvennye tradicii narodov Zapadnoj Sibiri (Traditions culturelles et économiques des peuples de Sibérie occidentale), Novosibirsk, 1989, pp. 133-140 ; I. V. Belič, V. B. Bogomolov, «Pogrebal'nyj ritual kurdakskosargatskih tatar » (Le rituel de l'inhumation chez les Tatars de Kurdak et Sargat), Eksperimental'naja arheologija (Tobolsk), 1, 1991, pp. 158-178; F. T. Valeev, Sibirskie tatary : kul'tura $i$ byt (Les Tatars de Sibérie : culture et mode de vie), Kazan, 1992, pp. 149-205, passim.

2. Voir par exemple S. A. Tokarev, Etnografija narodov SSSR (Ethnographie des peuples de l'URSS), Moscou, 1958, pp. 444-445.

3. F. T. Valeev, op. cit.,pp. 171-172.

4. V. V. Bartol'd, « Baraba », dans id., Sočinenija (Euvres), Moscou, 1965, 3, p. 366.

5. B. V. Mel'nikov, «Pozdnie pogrebal'nye pamjatniki taežnogo Priirtyša » (Les monuments funéraires tardifs des régions de taïga sur le cours du Moyen-Irtysh), in Drevnie pogrebanija Ob'-Irtyša (Sites funéraires anciens de l'Ob et de l'Irtysh), Omsk, 1991, p. 153. 
Tous les auteurs s'accordent, en tout cas, sur le fait que la diffusion de l'islam fut un processus lent, contradictoire et non dépourvu d'une certaine dimension dramatique et qu'il est donc impossible de rattacher ce phénomène à un événement historique isolé. L'importance accordée dans un certain nombre d'études à la date de 1394-1395 est due, en fait, à la fortune critique exceptionnelle qu'ont connue deux documents anciens conservés au Musée d'État de Tobolsk. Il s'agit de deux manuscrits rédigés en tatar, respectivement, par Sa'd Waqqâs Allâqulov et Kaššâf Abû-Sa‘îdov, publiés et traduits en russe au début du XXe siècle par Nikolaj F. Katanov ${ }^{6}$. Comme plusieurs travaux l'ont démontré depuis, il s'agit en fait de compilations de sources plus anciennes. Selon ces documents, l'an $797 \mathrm{de}$ l'hégire (1394-1395) vit l'arrivée de 366 šay $\underline{h}$ à cheval et de leur allié le khan Šaybân, suivi de 1700 guerriers d'élite, sur les berges de l'Irtysh où ils se lancèrent dans un grand combat contre les païens qui habitaient la région, les Khotan, Qara-Qiptchak et autres Nogaïs. Ces propagateurs de l'islam se présentaient

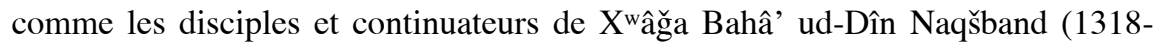
1389), l'une des figures clés de la grande confrérie soufie qui devait bientôt prendre son nom, la Naqšbandiyya.

Ces sources ont un caractère légendaire marqué. Leur lien étroit avec la tradition mythologique populaire apparaît, par exemple, dans le nombre de šay $\underline{h}$ dont ces textes font état et qui coïncide pratiquement avec le nombre de jours de l'année : selon un trait particulier à la vision du monde traditionnelle, des phénomènes d'ordre naturel ou cosmique sont saisis en un tout indivis avec les événements fondateurs de la vie d'une communauté humaine. (Rappelons-nous les douze apôtres - nombre correspondant à celui des mois de l'année - de la tradition mythologique chrétienne.)

Toujours est-il que les légendes relatives à la guerre sainte entreprise sur les berges de l'Irtysh sont encore vivantes aujourd'hui dans la mémoire populaire. Ainsi, on m'a raconté dans le village de Bajševo (district de Vagaj, dans la région de Tioumen) cette même histoire de 366 šay $\underline{h}$-prêcheurs venus d'Arabie combattre pour la foi islamique. Selon cette légende, tous furent tués par traîtrise, puis enterrés avec révérence par leurs fidèles, dans des cimetières-sanctuaires particuliers, appelés par la suite ostan. Les habitants de Bajševo pouvaient même désigner par son nom (Hakîm-atâ) le prêcheur enterré dans l'ostan voisin de leur localité. L'un des noms de famille répandus dans le village, celui de Šihov, dérivé du terme arabe šay $\underline{h}$, atteste lui aussi cette longue mémoire des premiers combats pour l'islam menés sur l'Irtysh.

Le village de Vtorovagaj (district de Vagaj, dans la même région de Tioumen) a conservé, lui, le souvenir de 360 šay $\underline{h}$ venus jadis de Boukhara (lieu de naissance de Bahâ' ud-Dîn Naqšband). Selon une étymologie populaire, même le nom du village

6. N. F. Katanov, « O religioznyh voinah šejha Bagauddina protiv inorodcev Zapadnoj Sibiri » (Des guerres religieuses du šay $\underline{h}$ Bahâ' ud-Dîn contre les allogènes de Sibérie occidentale), Ežegodnik Tobol'skogo gubernskogo muzeja, 14, 1905, pp. 1-28. 
de Čikča (ou Šykča, district de Tioumen) provient du mot šyk - forme dérivée de

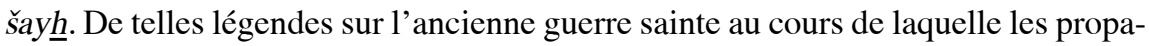
gateurs de l'islam détruisirent les vieilles idoles, kurčak, se sont conservées dans presque tous les groupes de Tatars de Sibérie.

Le caractère légendaire lui-même de nos sources explique le recours à la figure de Bahâ' ud-Dîn Naqšband, l'une des figures les plus éminentes du soufisme centrasiatique. Le personnage de Bahâ' ud-Dîn, extraordinairement populaire en Transoxiane, a pu séduire par ses origines modestes, dans la mesure où ce saint personnage était issu du milieu artisanal des bazars (naqšband : ciseleur de pochoirs de bois pour l'impression des tissus), et par le souvenir de sa vie d'ascèse. La légende relative à l'expédition des šay $\underline{h}$ a peut-être été élaborée dans un milieu influencé par la Naqšbandiyya, qui est devenue dès le XVe siècle, avec la Qâdiriyya, l'une des deux confréries (tarîqât) les plus influentes d'Asie Centrale. Or on sait à quel point la Naqšbandiyya s'est toujours distinguée par son très haut niveau d'activisme social, voire politique. Les membres de la tarîqa n'ont-ils pas joué un rôle déterminant pour l'enracinement de l'islam dans toute l'Asie Centrale, depuis les cités-oasis de Transoxiane ou de l'Altishahr jusqu'aux confédérations tribales qazaques et qyrghyzes?

L'activité de propagation de la foi des prêcheurs les plus dynamiques d'Asie Centrale s'est étendue en Sibérie dès avant le XIV e siècle, pour atteindre un point culminant aux $X^{\mathrm{e}}$ et $\mathrm{XVI}^{\mathrm{e}}$ siècles. Il est tout à fait possible que son renforcement soit dû à l'action de tarîqât particulières ou de branches distinctes de ces tarîqât. La guerre sainte plus ou moins imaginée dont il est question dans les sources manuscrites mentionnées plus haut ne constitue qu'un épisode dans le processus complexe de la conversion à l'islam des populations türkophones de Sibérie occidentale. Dans cette mesure, il me paraît impossible de parler d'une quelconque date concrète de pénétration de l'islam en Sibérie - un phénomène qu'il convient plutôt de rattacher au processus plus général d'enracinement de l'islam dans les régions les plus diverses de la vaste Asie Centrale.

La propagation de l'islam chez les Tatars et les Qazaqs de Sibérie présupposait un certain nombre de bouleversements significatifs dans leurs structures communautaires et politiques. C'est aux XVe et $\mathrm{XVI}^{\mathrm{e}}$ siècles, en effet, que l'on assiste à la constitution du premier noyau du peuple qazaq, dans le cadre du khanat qazaq alors en formation. Dans la seconde moitié du XVI siècle, c'est au tour du khanat de Sibérie de se transformer en un État indépendant. À cette époque, on assiste également à la formation des groupes ethniques tatars distincts. La propagation de l'islam nous apparaît donc rétrospectivement comme une expression parmi d'autres de ces processus sociaux et ethniques.

Comme tout autre système confessionnel, l'islam s'est développé parmi des populations chez lesquelles existait déjà le substrat de sa diffusion - en l'occurrence une construction communautaire avancée. Ainsi la formation des Tatars de Baraba en communauté ethnique distincte, autonome, se produit au cours du $\mathrm{XVII}^{\mathrm{e}}$ siècle et dans la première moitié du XVIII ${ }^{\mathrm{e}}$. Or si les Tatars de Baraba sont encore chamanistes au début du XVIII ${ }^{\mathrm{e}}$ siècle, l'islam se renforce parmi eux d'une 
manière extraordinairement rapide dès le milieu de ce siècle ${ }^{7}$. Par contre, dans le même temps, il est intéressant de noter que chez les Türks de la Čulym, une petite formation ethnique türke de Sibérie occidentale, le chamanisme est resté en vigueur sans se mêler d'emprunts ni chrétiens, ni islamiques ${ }^{8}$.

Parmi les Qazaqs, c'est pendant la première moitié du XVIII' siècle que l'islam trouva sa diffusion la plus large. Cet événement coïncida avec le début du rattachement à la Russie des tribus de la Horde médiane, dont les pacages se trouvaient sur le territoire de la Sibérie occidentale. L'influence islamique se renforça au cours du siècle suivant, au point que le célèbre savant qazaq Čokan Valihanov pouvait remarquer :

«Sous l'influence des mollahs tatars, des îšân d'Asie Médiane et de leurs prosélytes, la nation [qazaque] adopte de plus en plus un profil musulman commun : un certain nombre de sultans et de riches [qazaqs] enferment leurs femmes dans des yourtes séparées, comme au harem; des [Qazaqs] pieux commencent à se rendre à La Mecque, tandis que nos bardes, au lieu de chants populaires, récitent maintenant des apocryphes islamiques remodelés sous la forme de poèmes populaires. $»^{9}$

Il convient cependant de remarquer que les peuples de Sibérie ont pu prendre connaissance des religions universelles bien des siècles avant la pénétration de l'islam. Un rôle important, dans ces contacts, a pu être joué par les différentes formations étatiques du haut Moyen Âge comme le premier et le second kaghanats vieux-türks (552-630 et 682-745), le kaghanat ouïghour (745-840) et la confédération des Qyrghyz du Iénisseï ( $\mathrm{VI}^{\mathrm{e}}-\mathrm{XIII}{ }^{\mathrm{e}}$ siècles). Si l'idéologie principale de ces kaghanats turcs anciens était le chamanisme, il n'en reste pas moins que le manichéisme - synthèse de zoroastrisme, de bouddhisme et de christianisme nestorien - devint religion officielle de la confédération ouïghoure des peuples d'Asie Centrale et de Sibérie du sud, puis à l'époque des anciens Qyrghyz. (On connaît par ailleurs l'importance de l'influence nestorienne parmi les peuples de Haute Asie, en particulier chez les empereurs mongols, d'où la fameuse légende sur l'empire chrétien extrême-oriental du Prêtre Jean.)

Après l'éclatement de ces entités politiques, les groupes ethniques distincts qui les constituaient migrèrent souvent vers des régions fort éloignées de leur territoire initial. On a ainsi conservé des témoignages affirmant qu'une partie des Ouïghours, après leur défaite et leur massacre par les Qyrghyz de l’Iénisseï en 840,

7. Cf. A. G. Seleznev, Barabinskie tatary : istoki etnosa i kul'tury (Les Tatars de Baraba : les origines d'une ethnie et d'une culture), Novosibirsk, 1994, passim.

8. N. A. Tomilov, « Čulymcy », in V. A. Tiškov, ed., Narody Rossii. Enciklopedija (Les peuples de Russie. Encyclopédie), Moscou, Naučnoe izdatel'stvo Bol’šaja Rossijskaja Enciklopedija, 1994,pp. 410-412, ill. (n.d.t.).

9. Č. Č. Valihanov, «Sledy šamanstva u kirgizov» (Les reliquats de chamanisme chez les Qazaqs), in id., Sočinenija (Euvres), Alma-Ata, 1985, 4, p. 49. 
« s'installèrent dans les forêts de l'Irtysh, renonçant à l'élevage au profit de la pêche et de la chasse à la loutre, à la zibeline, à la martre et au lièvre, pour se nourrir de leur viande et se vêtir de leurs peaux; de sorte qu'ils ne virent plus jamais ni bétail, ni toile de lin, ni tissus de coton... »10

On peut ainsi supposer que les populations manichéennes qui faisaient partie du kaghanat ouïghour se retrouvèrent dans les régions qui virent quelques siècles plus tard se dérouler le drame décrit dans les manuscrits de Tobolsk et dont la mémoire populaire a si longtemps conservé le souvenir. Nous ne pouvons exclure qu'une partie de ces Ouïghours se soient intégrés à l'entité ethno-politique des KimakQiptchaqs, apparue sur l'Irtysh au IX ${ }^{\mathrm{e}}$ siècle. Rappelons que l'un des peuples parmi lesquels la légende des šay $\underline{h}$ est restée le plus active est celui des Qara-Qiptchaqs. Le second peuple mentionné dans nos manuscrits, celui des Khotans, est lui aussi d'un grand intérêt pour notre propos : le peuple khotan est en effet constitué de descendants de ces Qara-Khitays qui, en 1141, taillèrent en pièces les Seldjoukides au cours d'une des plus grandes défaites musulmanes de l'histoire. Jusqu'à nos jours, les Khantes et les Selkoupes ${ }^{11}$ désignent les populations tatares voisines de leurs propres localités sous le nom de Tatar-Katan-Yakhs (katan et khotan apparaissant comme une forme dérivée de kitay ou khitay) ${ }^{12}$.

Les peuples de Sibérie apparaissent donc, dès une époque ancienne, familiers des échanges avec les formes les plus diverses de religion, depuis les plus « primitives » jusqu'aux plus « développées » et cette expérience se manifeste d'une manière particulièrement évidente dans l'ethnographie des musulmans de Sibérie. Cependant c'est sans doute le chamanisme qui a laissé la trace la plus profonde dans leur héritage culturel. Du reste les conceptions religieuses préislamiques chez les Tatars et les Qazaqs de Sibérie ont déjà fait l'objet de très nombreuses études.

Les Qazaqs et presque tous les groupes tatars ont conservé le nom Tengre avec le sens de « dieu » ou de « divinité ». Le même mot servait à désigner la divinité suprême à l'époque des kaghanats türks anciens, de même que chez les populations chamanistes de l'Altaï. Mais les Tatars et les Qazaqs de Sibérie connaissent aussi d'autres esprits et divinités que vénéraient jadis les Türks et les Mongols dans le cadre du chamanisme - tels Yer-Su (litt. « Terre-Eau »), Umay, Qut, Natigay, peut-être aussi Aurak-arvah. Les Qazaqs de la région d'Omsk emploient souvent l'expression Qutty bolsyn! : « plaise à Dieu ». C'est par contre à travers l'influence des cultures antiques et médiévales d'Asie médiane que l'on peut expliquer la préservation par les Qazaqs et les Tatars d'un culte du feu, de même que la présence du terme d'origine persane kuday, avec le sens de « dieu », dans la langue des Qazaqs et dans celle de presque tous

10. V. V. Radlov, «K voprosu ob ujgurah » (À propos du problème des Ouïghours), Zapiski Imperatorskoj Akademii nauk, 62, 1893, annexe (priloženie) 2, p. 55.

11. Sur ces deux groupes ethniques d'inégale importance, voir : Z. P. Sokolova, « Hanty », in Narody Rossii..., op. cit., pp. 380-382, ill. ; V. I. Vasil'ev, «Sel'kupy », ibid., pp. 312-314 (n. d.t.).

12. G. I. Pelih, Sel'kupy XVII veka. Očerki social'no-ekonomičeskoj istorii (Les Selkoupes au XVII siècle. Études d'histoire sociale et économique), Novosibirsk, 1981, pp. 59-60. 
les groupes de Tatars de Sibérie. (Notons que le même mot se rencontre également parmi les populations chamanistes de l'Altaii.)

De très anciennes conceptions chamanistiques sont sans doute à l'origine de représentations d'essence animiste, exprimées notamment sous la forme d'un culte cynégétique des esprits du lieu (uyas, ese) : maîtres d'un cours d'eau (su uyasy), d'une forêt (pičin, pitsin, urman uyasy), d'une maison (oy uyasy), etc. ${ }^{13}$. C'est chez les Tatars de Baraba que les représentations chamanistes ont été préservées de la manière la plus complète. Rappelons que ces derniers figurent parmi les quelques groupes ethniques türkophones de la plaine de Sibérie occidentale qui ont conservé, jusqu'au début du XVIII siècle, une vision du monde et des pratiques cultuelles essentiellement chamanistes. La propagation de l'islam chez les Tatars de Baraba ne date que de la seconde moitié du XVIII ${ }^{\mathrm{e}}$ siècle, bien qu'un petit nombre de musulmans soit mentionné à Baraba dès les premières années de ce siècle ${ }^{14}$. Cette circonstance a attiré l'intérêt de nombreux chercheurs pour les conceptions religieuses préislamiques des aborigènes de Baraba ${ }^{15}$.

13. Voir entre autres H. A. Argynbaev, Sem'ja i brak u kazahov (La famille et le mariage chez les Qazaqs), Alma-Ata, 1975, passim ; Š. K. Ahmetova, « Tradicionnaja pogrebal'naja obrjadnost' kazahov g. Omska » (Le rite funéraire traditionnel des Qazaqs d'Omsk), in Etničeskaja istorija tjurkskih narodov Sibiri i sopredel'nyh territorij (po dannym etnografii) (L'histoire ethnique des peuples türks de Sibérie et des territoires limitrophes (d'après les données de l'ethnographie)), Omsk, 1992, pp. 13-16; Z. A. Baširova, «Priroda v religioznyh predstavlenijah sibirskih tatar» (La nature dans les représentations religieuses des Tatars de Sibérie), in Etničeskaja istorija tjurkojazyčnyh narodov Sibiri i sopredel'nyh territorij ( $L$ 'histoire ethnique des peuples türkophones de Sibérie et des territoires limitrophes), Omsk, 1984, pp. 87-89 ; I. V. Belič, op. cit., pp. 133-140 ; F. T. Valeev, op. cit., pp. 177-192 ; id., O religioznyh predstavlenijah narodov Sibiri i Severa (Des représentations religieuses des peuples de Sibérie et du Grand Nord), Leningrad, 1976, pp. 320-331 ; R. M. Mustafina, Predstavlenija, kul'ty, obrjady u kazahov (Représentations, cultes et rites chez les Qazaqs), Alma-Ata, 1992, pp. 126-152 ; A. T. Toleubaev, Relikty domusul'manskih verovanij v semejnoj obrjadnosti kazahov (Reliquats de croyances préislamiques dans les rituels familiaux des Qazaqs), Alma-Ata, 1991, passim; N. A. Tomilov, Očerki etnografii tjurkskogo naselenija Tomskogo Priob'ja, (Études d'ethnographie de la population türke de l'Ob, dans la région de Tomsk), Tomsk, 1983, pp. 174-184 ; K. S. Šulembaev, Magi, bogi i dejstvitel'nost' (Mages, dieux et réalité), Alma-Ata, 1975, p. 23 ; M. B. Fateeva, «Religioznye verovanija sibirskih tatar: obrazy duhov-hozjaev» (Croyances religieuses des Tatars de Sibérie : les images d'esprits du lieu), in Problemy etnografii i sociologii kul'tury (Problèmes d'ethnographie et de sociologie de la culture), Omsk, 1988,pp. 106-107.

14. Ja. I. Lindenau, Opisanie narodov Sibiri (Description des peuples de Sibérie), Magadan, 1983, pp. 148-150.

15. Cf. I. V. Belič, op. cit., pp. 133-140 ; V. Diószegi, « Pre-Islamic shamanism of the Baraba Turks and some ethnogenetic conclusions », in Shamanism in Siberia, Budapest, 1978, pp. 83167 ; E. Lot-Falck, « Les tambours shamaniques des Türks barabines : étude comparée », Ethnographie, n. sér., 67, 1973, pp. 18-46 ; S. V. Ivanov, Skul'ptura altajcev, hakasov i sibirskih tatar (La sculpture des Altaïens, des Khakasses et des Tatars de Sibérie), Leningrad, 1979, pp. 143-145 ; Ja. I. Lindenau, op. cit., pp. 148-150 ; L. P. Potapov, Altajskij šamanizm (Le chamanisme altaïen), Leningrad, 1991, pp. 183-184 ; V. Radlov, Sibirskie drevnosti (Antiquités sibériennes), Saint-Pétersbourg, 1891, I, 2e partie, p. 33, tabl. VI ; A. G. Seleznev, « K semantike šamanskih atributov u tjurkojazyčnyh narodov Sibiri » (Contribution à la sémantique des attributs chamaniques chez les peuples türkophones de Sibérie), in Etničeskie i social'nokul'turnye processy u narodov SSSR (Processus ethniques et socio-culturels chez les peuples de l'URSS), Omsk, 1990, 2, passim ; id., « K harakteristike šamanskih atributov barabinskih tatar » (Contribution à la typologie des attributs chamaniques chez les Tatars de Baraba), in 
On trouve notamment, dans les sources de la fin du XVII siècle et du début du $\mathrm{XVIII}^{\mathrm{e}}$, des descriptions détaillées de chamanes de Baraba (kam), des attributs du culte chamanique et des rites chamaniques eux-mêmes. La tentative la plus complète d'étude du chamanisme barabien est due à Vilmos Diószegi ${ }^{16}$, qui a tiré un grand parti de ces sources manuscrites en analysant chaque élément dans ses relations avec telle ou telle communauté ethno-culturelle. L'ethnographe hongrois a ainsi réussi à mettre en lumière trois groupes de phénomènes apparentés. Le premier groupe est celui des phénomènes contenant des attributs spécifiques du chamanisme (tels que la fabrication du tambourin du chamane, les pratiques rituelles bruyantes, ou encore les idoles chamaniques) et dont on ne trouve d'analogie que chez les peuples de Sibérie méridionale, en l'occurrence chez les Türks de l'Altaï. Le second groupe réunit des sacrifices d'animaux, des funérailles aériennes ou la divination par le tir à l'arc, toutes pratiques qui se rencontrent chez les peuples aussi bien ouraliens que türks de Sibérie, et souvent même bien au-delà de cette région. Le troisième groupe est celui de pratiques que l'on ne rencontre qu'en milieu ouralien, à savoir la conservation d'idoles anthropomorphiques de bois dans des étuis spécialement conçus à cet effet, la tradition du transport de ces idoles sur de petits traîneaux réservés à cet usage et le rituel consistant à préparer des effigies à tête pointue.

Ces informations manuscrites sur la conservation, par les gens de Baraba, de leurs idoles dans des boîtes spéciales sont confirmées par les dires de mes informateurs. Ces derniers m'ont raconté que l'on conservait ces effigies anthropomorphiques de bois (ou de tissu) soit dans les maisons, soit à l'extérieur des villages, au creux de bosquets sacrés. Les idoles étaient déposées dans de petites fosses ou bien dans des édicules construits à leur intention. Les anciens se souviennent que ces « poupées » (qurčak) étaient nourries, qu'on leur demandait la permission de se rendre à la chasse, qu'on les remerciait ensuite en cas de succès, etc. Selon le témoignage d'un ambassadeur russe de passage en 1692, les gens de Baraba offraient à leurs idoles le meilleur du produit de leur chasse ${ }^{17}$.

On trouve des informations analogues sur les idoles (kaurčak, kamurčak) des Tatars de Baraba chez Vladimir Bogomolov. Selon ce dernier, dans la seconde moitié du XIXe siècle les boîtes ou étuis à idoles étaient tombés en désuétude, mais

\footnotetext{
Šamanizm kak religija : genezis, rekonstrukcija i tradicii (Le chamanisme comme religion : genèse, reconstruction et traditions), Yakoutsk, 1992, passim ; Z. D. Titova, Istočniki XVIII V. na zapadnoevropejskih jazykah po izučeniju korennyh narodov Sibiri i Severo-Vostoka. Dissertacija (Les sources du XVIII siècle en langues européennes occidentales pour l'étude des peuples autochtones de Sibérie et du Nord-Est. Thèse), Leningrad, 1989, pp. 183-184, 187, $349,352-353,425-429$ et 717.

16. V. Diószegi, op. cit., pp. 83-167.

17. «Putešestvie i žurnal po ukazu Velikih Gosudarej, Carej i Velikih Knjazej Ioanna Alekseeviča i Petra Alekseeviča otpravlennogo iz Moskvy v Kitaj, Gospodina Ebergarda Izbrannedesa Poslannikom v 1692 godu, Marta 14 dnja » (Voyage et journal sur l'ordre des Éminentissimes Souverains, Tsars et Grands Princes Ivan Alekseevič et Petr Alekseevič, du Seigneur Eberhard Izbrannedes, envoyé de Moscou en Chine comme Ambassadeur, le 14 mars 1692), Drevnjaja Rossijskaja Bibliografija, 1789, 9e partie, p. 437.
} 
l'on utilisait à leur place des ustensiles en bois de bouleau. On pratiquait la coutume du culte des idoles à la périphérie des villages, dans un creux pratiqué dans le tronc d'un bouleau sacré, ou bien sur ses branches : on nourissait ces idoles en leur offrant des sacrifices d'animaux, sous la forme d'aspersions de sang. On peut reconstituer leur aspect extérieur d'après les figures de la sculpture anthropomorphique pratiquée par les gens de Baraba : il s'agissait d'effigies de petite taille entre 10 et 15 centimètres seulement - , avec une ossature constituée de nattes de textile ou de cotonnade, sur lesquelles étaient endossés des «vêtements ». Bogomolov raconte que ces vêtements étaient tissés à partir d'un fragment de chaque morceau de tissu nouvellement acheté, et que leur forme reproduisait en miniature le costume traditionnel respectif des divers groupes de la population tuükophone de la plaine de Sibérie occidentale ${ }^{18}$.

Il est manifeste que ces poupées kurčak de Baraba (comme du reste celles que l'on trouve chez toutes les populations tatares de Sibérie) possèdent une pluralité de sens et de fonctions : elles apparaissent, d'une part, comme un symbole des mânes ancestrales - ces dernières incluant celles des aïeux du chamane - , et d'autre part comme des images des divinités protectrices, claniques mais aussi familiales. Avec le temps, ces deux hypostases (ou davantage...) ont fini par se confondre pour apparaître comme une entité indivise. C'est sous cette forme qu'elles ont été décrites par les premiers observateurs des Tatars de Baraba et il n'en est que plus difficile de faire maintenant la part de leurs composantes. Pour ce faire, il ne paraît pas déplacé de faire appel à des phénomènes analogues, au nord de la Sibérie (Oural) comme au sud.

Répandues chez tous les groupes ougriens de l'Ob, les niches à idoles sacrées abritaient des figures d'esprits ancestraux zoo- ou anthropomorphiques et elles étaient disposées, la plupart du temps, à la périphérie des villages, dans quelque endroit sacré. Des niches analogues existaient chez les Selkoupes. Parmi les populations türkophones de Sibérie, l'existence de niches abritant des effigies d'esprits ancestraux est attestée chez les Tatars dits «d'outre-marais » (zabolotnye) ou de Yaskolb. L'ethnographe Zoja Sokolova considère l'institution de ces lieux sacrés comme un phénomène extrêmement ancien et commun à l'ensemble des peuples ouraliens ${ }^{19}$.

Vilmos Diószegi a trouvé des analogies aux idoles à tête pointue de Baraba chez les Khantes (pugos), les Mansis (pubyh, pupi), les Selkoupes et les Nénetses. Dans les récits mythologiques des Ougriens de l'Ob, la tête pointue apparaît très souvent comme un attribut des géants mangeurs d'hommes. Si l'on se réfère à l'archéologie de Baraba, on remarque que des idoles de bois à tête pointue ont été mises au jour sur un site cultuel qui se trouve à l'intérieur du remarquable complexe de Sopka-2 (district de Venger, région de Novosibirsk). Ce site, daté du XIII ${ }^{\mathrm{e}}$ au XVIII ${ }^{\mathrm{e}}$ siècle de

18. V. B. Bogomolov, « Skul'ptura sibirskih tatar XIX-pervoj treti XX vv. » (La sculpture des Tatars de Sibérie au XIX ${ }^{\mathrm{e}}$ siècle et dans le premier tiers du XXe), in Etničeskaja istorija tjurkskih narodov..., op. cit., pp. 23-26.

19. Z. P. Sokolova, Social'naja organizacija hantov $i$ mansy V XVIII-XIX VV. (L'organisation sociale des Khantes et des Mansis auX XVIII e et XIX siècles), Moscou, 1983, p. 117. 
notre ère, a livré trois figurines : deux statuettes anthropomorphiques dotées d'une tête pointue, ainsi que de mains et de pieds; la troisième statuette, anthropozoomorphique, possède une queue semblable à celle d'un oiseau et un phallus, mais elle est aussi pourvue de la même tête à extrémité pointue ${ }^{20}$. Le sanctuaire relève sans aucun doute du cercle des cultures du Grand Nord et, selon ses inventeurs, appartenait à une population ougrienne.

On trouve des parallèles sud-sibériens (en particulier saïano-altaïques) au tungur, le tambourin des chamanes de Baraba ( $q a m)$, et aux idoles précédemment décrites, lesquelles agissent ici dans un rôle d'esprits auxiliaires du chamane. Si l'on en juge par les quelques illustrations qui nous en sont parvenues, le tambourin des chamanes de Baraba avait une forme circulaire, il était équipé d'un manche anthropomorphique et ne portait aucun dessin sur sa face supérieure ${ }^{21}$. Ainsi les figurines anthropomorphiques des Tatars de Baraba décrites ci-dessus apparaissent reliées sémantiquement avec les attributs du chamane, en particulier avec son tambourin.

Une source majeure, sur ce point, est constituée par la terminologie relative à ces idoles. En dépit de la grande rareté des matériaux touchant aux croyances des aborigènes de Baraba, j'ai pu identifier au cours de conversations avec les habitants du lieu trois termes désignant les figurines anthropomorphiques de bois ou de tissu : qurčak, čalu, etteney. On sait que les Tatars de Tobolsk employaient le nom Attaney pour désigner leur divinité suprême. Mes informateurs dérivent avec certitude ce nom Attaney du turc ata, « le père » (ce qui suggère, en passant, une claire perception de ces figurines comme symboles de l'esprit des ancêtres). Dans un certain nombre de localités - notamment le village de Tukuz, district de Vagaj, région de Tioumen - , le même nom est utilisé en même temps que celui de Kuday, commun chez les Turcs de Sibérie occidentale et méridionale, pour signifier « dieu » ou « divinité ». Selon Foat Väleev, le terme ettenay désignait chez certains groupes de Tatars de Sibérie de grandes figures de bois représentant l'esprit du clan, auxquelles on apportait des victimes ${ }^{22}$. La parenté du terme barabien etteney et du mot tatar sibérien ettenay ou attaney ne fait pas de doute.

Les figurines anthropomorphiques désignées sous le terme de kurčak étaient utilisées pratiquement par tous les groupes de Tatars de Sibérie. L'analyse étymologique, par Vilmos Diószegi, de ce terme de qurčak révèle le lien entre ce dernier et le tambourin du chamane, ainsi que d'autres attributs chamaniques

20. V. I. Molodin, «Kul'tovye pamjatniki ugorskogo naselenija lesostepnogo Ob'-Irtyša » (Monuments cultuels de la population ougrienne de l'Ob-Irtysh steppique et forestier), in Mirovozzrenie finno-ugorskih narodov (La vision du monde des peuples finno-ougriens), Novosibirsk, 1990, pp. 128-140, ill. 6 et 7 ; V. I. Molodin, V. I. Sobolev, A. I. Solov'ev, Baraba v epohu pozdnego srednevekov'ja (Baraba à l'époque médiévale tardive), Novosibirsk, 1990, pp. 177-183.

21.S. V. Ivanov, Skul'ptura altajcev, hakasov i sibirskih tatar..,, op. cit., fig. 151 ; Ph. I. Strahlenberg, Das nord-und ostliche Theil von Europa und Asia, Stockholm, 1730, tab. VI.

22. Cité par S. V. Ivanov, op. cit., pp. 143-144. 
communs aux peuples turcs de Sibérie méridionale : si qurčak signifie «idole » en sibéro-tatar, le même terme sert en altaïque et en sagaïque à désigner l'armature circulaire du tambourin du chamane; la forme qurču signifie en téléoute «cercle», tandis qu'elle désigne en altaïque les anneaux tintunabulants qui ornent le costume du chamane ${ }^{23}$.

Le terme le plus répandu dans l'Altaï est celui de čalu, qui désignait les instruments de culte les plus divers. On sait en particulier que ce mot servait à désigner le tambourin des chamanes de l'Altaï. Dans son étude capitale sur le chamanisme dans cette région, le vénérable ethnologue russe Leonid Potapov distingue trois types de tambourin, dont le principal élément de différenciation est constitué par le manche de bois symbolisant le propriétaire de l'instrument (čalu aazy) : l'un appelé mars čalu, un second appelé aki baštu čalu (čalu à deux têtes) et un troisième, ğangys bašty čalu: čalu à une seule tête. Le tambour de chamane des Tatars de Baraba se rapproche le plus du type de tambourin dont le manche est exécuté sous cette troisième forme de figure anthropomorphique à une seule tête. Outre les Tatars de Baraba, le même type de tambourin se rencontre encore chez les Telengoutes, les Telesses, les Téléoutes du Haut-Altaï et, au-delà des frontières de l'Altaï, chez les Touvines de Kobda ${ }^{24}$.

Selon le témoignage de Jakob Lindenau, un membre de la première expédition académique russe, entreprise dans les années 1730-1740, les kam de Baraba chamanisaient au XVIII ${ }^{\mathrm{e}}$ siècle en s'aidant d'un tambourin appelé tungur «au milieu » duquel se trouvait la représentation d'une idole - ğoese [ou iyase, le maître du tambour]. Les kam de Baraba connaissaient également le terme čalu: selon Lindenau, c'est ainsi que l'on appelait l'esprit-devin par le truchement duquel le chamane apprenait « beaucoup de choses inconnues $»^{25}$.

Ce lien entre les représentations anthropomorphiques et le tambour du chamane est confirmé par les matériaux dont nous disposons sur les populations altaïennes et téléoutes. Chez les premières, le terme čalu désignait soit le tambour lui-même, soit le manche du tambour figurant un ancêtre du chamane, maître de l'instrument, soit encore un petit tambourin, ou bien les nattes constituant l'habit du chamane (manyak), ou enfin des chiffons (yalama). Par ce même terme de čalu, les Téléoutes désignent le tambourin du chamane, le manche non anthropomorphique de ce même tambourin, ainsi qu'un ensemble d'instruments cultuels, parmi lesquels un modèle réduit du tambourin fabriqué à la mémoire des ancêtres de ce dernier, ou encore des représentations anthropomorphiques également désignées par les termes de kurmuš et d'amagander. Les observations de Johann P. Falk confirment que les

23. V. Diószegi, op. cit.,p. 135 ; D. A. Funk, Bačatskie teleuty v XVIII-pervoj četverti XX veka : istoriko-etnografičeskoe issledovanie (Les Téléoutes du Batchat au XVIII siècle et dans le premier quart du XIX $X^{\mathrm{e}}$ : étude historique et ethnographique), Moscou, 1993, p. 226.

24. L. P. Potapov, op. cit., pp. 183-184.

25. Ja. I. Lindenau, op. cit., pp. 148-149. 
idoles téléoutes «tschalu » étaient représentées sous la forme de créatures anthropomorphiques $^{26}$.

Le matériau lexicographique confirme cette hypothèse du lien entre les tambourins et les idoles anthropomorphiques. Le premier à démontrer ce lien fut le turcologue russe Vasilij Radlov, dans sa critique de l'ouvrage d'Andrej Anohin, Materialy po šamanstvu u altajcev (Matériaux sur le chamanisme chez les Altaïens) (1924). Attardons-nous un peu sur ces éléments lexicographiques, tels qu'ils apparaissent dans le dictionnaire de Radlov : čal (en ouïghour, ottoman, tatar de Crimée, karaime, tatar de Kazan) : frapper d'un coup, jeter, jouer d'un instrument de musique; čalyp (en ottoman, karaime et tatar de Kazan) : être battu, être ce dont on joue; čalu (en altaïque) : tambour du chamane, idole ${ }^{27}$. De sorte que c'est toute une ligne sémantique qui est ici constituée par les termes servant à désigner à la fois un instrument de musique à percussion (le tambourin du chamane) et des figures anthropomorphiques.

Cet ensemble de données, qui atteste les relations génétiques entre chamanismes barabien et altaïen, va nous permettre d'identifier les sources de représentations chamanistiques communes. Dans l'Altaï, les figures anthropomorphiques constituant le manche du tambourin représentent à la fois le « maître » de ce tambourin, le « maître » d'une localité déterminée et le protecteur de la chasse. On retrouve les mêmes fonctions chez les Tatars de Baraba. Dans la cosmographie traditionnelle des gens de Baraba, une place importante était dévolue aux représentations liées au cultes cynégétiques, dans le cadre desquels les esprits maîtres des lieux et des éléments jouaient un rôle significatif : l'esprit de l'eau (su iyasy), de la terre (yariyasy), du logis (uy-iyasy), de la forêt (bičen, urman-iyasy), etc. Le lien entre les figures anthropomorphiques appelées čalu ou kurčak s'exprimait dans le rituel de l'alimentation de l'idole avec le sang des bêtes capturées à la chasse.

Les représentations de la figure du manche comme du maître du tambourin sont confirmées par cette indication donnée par Jakob Lindenau, selon laquelle l'idole figurant sur le tambourin de Baraba était appelée « ğoese » (iyase). Ainsi il apparaît que cette strate commune aux représentations chamanistiques des Barabiens et des Türks de l'Altaï a été constituée dans un milieu de chasseur à une époque extrêmement ancienne.

26. V. Verbickij, Slovar' altajskogo i aladagskogo narečij tjurkskogo jazyka (Dictionnaire des parlers altaïques et aladagiques de la langue türke), Kazan, 1884, p. 422 ; N. P. Dyren'kova, «Materialy po šamanstvu u teleutov» (Matériaux sur le chamanisme chez les Téléoutes), Sbornik Muzeja antropologii i etnografii, 10, 1949, pp. 112, 187-190 ; S. V. Ivanov, Skul'ptura altajcev..., op. cit., pp. 100-101 ; L. P. Potapov, « Buben teleutskoj šamanki i ego risunki » (Le tambourin d'une chamane téléoute et ses dessins), Sbornik Muzeja antropologii i etnografii, 10, 1949, p. 192 ; L. P. Potapow, « Die Schamanentrommel bei den Altaischen Völkerschaften », in Glaubenswelt und Folklore der Sibirischen Völker, Budapest, 1963, p. 228 ; Z. D. Titova, op. cit., pp. 686-687.

27. A. V. Anohin, « Materialy po šamanstvu u altajcev » (Matériaux sur le chamanisme chez les Altaïens), Sbornik Muzeja antropologii i etnografii, 4, 2, 1924, p. 49 ; V. Radlov, Opyt slovarja tjurkskih narečij (Essai de dictionnaire des dialectes türks), Saint-Pétersbourg, 1900, 3, col. 1875, 1876, 1881, 1884 . 
La symbiose islam-chamanisme pénètre pratiquement tous les aspects du mode de vie des Tatars de Sibérie. À titre d'exemple, on peut évoquer le rite funéraire. D'un côté, ce rituel comporte une orientation islamique manifeste : les obsèques ont lieu en présence d'un mollah, elles s'accompagnent de la lecture de nombreuses prières musulmanes, des symboles islamiques tels que les croissants de lune sont ensuite dressés sur la tombe du défunt, lequel est déposé, enveloppé d'un simple linceul, dans une fosse que l'on a pris soin de creuser en ménageant une niche latérale; l'interdit de la présence des femmes dans le cortège funéraire et au cimetière est respecté scrupuleusement.

Mais dans le même temps, cet ensemble de rites se mêle de pratiques chamaniques. Ainsi, dans certains groupes de Tatars de Sibérie, le double non corporel matérialisé du défunt, qui apparaît trois ou quatre jours après le décès, porte le nom de qut ou de yulo.

On sait que le terme de kut, servant à désigner le double intérieur de l'être humain, symbolisant sont hypostase existentielle, est répandu d'une manière extraordinairement large dans les langues türkes de l'Altaï : chez les Téléoutes, les Tchelkanes, les Chors, les Kumandis, les Katchines, les Sagays, les Beltires, les Touvines, ainsi que chez les Yakoutes, les Dolganes, les Qyrghyz et les Tatars de Sibérie, sans oublier les Kètes, chez lesquels le même mot est l'un de ceux utilisés pour désigner le chamane. Ce terme de kut apparaît souvent dans les sources manuscrites türkes médiévales, notamment celles écrites en caractères runiques, et il entrait dans le fonds lexical prototürk.

Tout aussi important, dans la pratique religieuse du chamanisme altaïen, le concept de double intérieur de l'homme ordinaire comme du chamane était aussi désigné sous le terme de ğula (dyula, čula, etc., équivalent de yulo chez les Tatars sibériens du Tobol et de l'Irtysh). C'est précisément le ğula kama qui voyage dans les cieux lorsqu'opère le chamane, tandis que son enveloppe terrestre demeure visible de tous.

Il est en tout cas révélateur que, chez les Telengoutes et les Teles, le vieux mot de $k u t$, avec le sens de «double », ait été supplanté par celui de ğula - un terme doté, du reste, d'une ancienneté pour le moins millénaire, puisqu'il apparaît pour la première fois chez Mahmûd Kâshgharî. Chez certains autres groupes ethniques, les deux termes voisinent comme synonymes ${ }^{28}$. Un phénomène analogue de substitution d'un terme par un autre synonyme semble s'être produit parmi les Tatars de Sibérie. Chez ces derniers, cependant, la strate türke ancienne de leur terminologie a accueilli des termes chargés d'éléments islamiques, tels que les mots awrak et čan, d'origine arabo-persane.

Des convictions chamanistes continuent néanmoins de se refléter sur les monuments funéraires des Tatars de Sibérie - poteaux (kirtme, sukky, oy) ou stèles

28. Pour nous limiter aux travaux les plus sérieux sur ces questions, mentionnons: A. V. Anohin, op. cit., pp. 19-21 ; I. V. Belič, «Avrak », in Narody Sibiri i sopredel'nyh territorij (Les peuples de Sibérie et des territoires limitrophes), Tomsk, 1995, pp. 254-262 ; F. T. Valeev, Sibirskie tatary..., op. cit., pp. 180-181 ; N. P. Dyren'kova, art. cit., pp. 108-190 ; L. P. Potapov, Altajskij šamanizm, op. cit., pp. 33-53 ; D. A. Funk, op. cit., passim. 
(bagan, orma) dont on peut rencontrer, il va sans dire, de très anciennes analogies chez les peuples les plus divers. Une charge sémantique profonde et ancienne recouvre des stèles funéraires décorées avec éclat et destinées à frapper l'œil. L’idée la plus générale exprimée par ces monuments est sans aucun doute celle de verticalité, d'aspiration de la terre vers le ciel (et inversement), symbolisant le lien éternel et indesctructible de l'Univers. Dans ce sens, ils peuvent être identifés avec l' «Arbre du Monde », ou avec les longes qui constituent vraisemblablement son reflet symbolique. Un examen un peu plus détaillé de ces stèles m'a permis de découvrir, sur leur partie supérieure et médiane, des incisions transversales, effectuées sur tout le pourtour en rangs verticaux très légèrement obliques.

La charge sémantique forte de ces incisions nous est révélée par leur extrême fréquence et leur expressivité particulière, puisque une partie de ces anfractuosités était remplie de peinture rouge et se détachait donc très visiblement sur le fond nu de chaque stèle. J'ai pu découvrir leur signification en ayant recours à l'analyse comparative : dans le cas présent, il semble que l'on soit en face d'une analogie des « escaliers chamaniques » que les Tatars de Sibérie gravaient sous forme d'incisions (tapty, litt. « marches ») sur le tronc de leurs bouleaux sacrés.

De telles entailles étaient également pratiquées par les chamanes des Chors et des Altaïens, pendant leurs voyages dans le «Monde supérieur». Chez les Téléoutes du Batchat, ce terme tapty sert à désigner chacune des nombreuses couches de ce Monde supérieur. Le nombre d'intailles dépendait de la couche du ciel dans laquelle vivait l'esprit auquel le chamane rendait visite. Le mot tapty s'appliquait aussi bien à ces intailles qu'aux couches célestes elles-mêmes. Chez les Chors, la stèle portant des bandes alternées tracées à la peinture rouge était utilisée pour la cérémonie accompagnant le recouvrement de l'âme d'un malade par le chamane. Le représentation d'une lance plantée sur la tombe favorisait chez les Qazaqs l'envol vers le ciel de l'âme du mort. Dans l'imaginaire finnois, l'âmedouble du défunt atteignait le royaume des Manaly disparus en rampant sur la Montagne cosmique située dans le pays du Nord; le défunt progressait sur le flanc de la montagne en prenant appui sur des entailles qu'il y pratiquait avec les ongles. Chez les chamanes yakoutes, enfin, lors des voyages vers le monde supérieur l'élément de liaison était constitué par des longes (serge) particulières ${ }^{29}$.

29. Cf. V. N. Basilov, Šamanstvo u narodov Srednej Azii i Kazahstana (Le chamanisme chez les peuples d'Asie médiane et du Qazaqstan), Moscou, 1992, pp. 199-204 ; V. P. D'jakonova, « Tuvinskie šamany i ih social'naja rol' v obščestve » (Les chamanes de Touva et leur rôle dans la société), in Problemy istorii obščestvennogo soznanija aborigenov Sibiri (Problèmes d'histoire de la conscience communautaire des aborigènes de Sibérie), Leningrad, 1981, p. 162 ; S. V. Ivanov, « Jakutskie konovjazi » (Longes yakoutes), in Material'naja kul'tura narodov Sibiri i Severa (La culture matérielle des peuples de Sibérie et du Grand-Nord), Leningrad, 1976, p. 219 ; M. F. Kosarev, Zapadnaja Sibir'v drevnosti (La Sibérie occidentale dans l'antiquité), Moscou, 1984, p. 217 ; L. P. Kyzlasov, Drevnejšaja Hakasija (La Khakassie préhistorique), Moscou, 1986, p. 196 ; R. M. Mustafina, Predstavlenija, kul'ty, obrjady u kazahov v kontekste bytovogo islama v Južnom Kazahstane (Croyances, cultes et rites chez les Qazaqs, dans le contexte d'un islam coutumier, au Qazaqstan méridional), Alma-Ata, 1992, p. 97 ; C. C. Musi, Shamanism from East to West, Budapest, 1997, p. 92 ; D. A. Funk, op. cit., p. 203 ; V. F. Jakovlev, Serge, Yakoutsk, 1992, 1re partie, p. 11. 
Certes l'image de l'escalier constitue dans la mythologie des peuples les plus divers un instrument de communication privilégié entre les mondes, en particulier lorsque les forces cosmiques sont sollicitées pour l'établissement d'un ordre nouveau sur la terre. Des escaliers de chamane d'époque préhistorique, archétypes des marches-incisions figurant sur les stèles funéraires des Tatars du Tobol, ont été découverts sur les célèbres stèles et menhirs qui particularisent la culture du Bronze ancien en Sibérie méridionale. Ces escaliers apparaissent sous la forme de motifs en forme d'agrafes orientées vers le bas, pratiquées en grand nombre sur le bord oblique de ces stelles et représentant les traverses d'échelles de corde. À ce jour, il s'agit de la plus ancienne figuration, en Sibérie, de l'escalier de chamane pénétrant les différents mondes de l'Univers. De ces traverses semblent descendre toutes les incisions symbolisant les marches de l'escalier sur la voie céleste du chamane. À la base de ces représentations, on ne trouve ni plus ni moins que le mythologème de l'escalier comme instrument de passage entre les mondes, depuis celui sur lequel l'ancien dieu égyptien Seth monte vers le ciel, jusqu'à celui qui a donné son nom à la célèbre ballade $A$ stairway for heaven ${ }^{30}$.

Les Tatars du Tobol apposent parfois sur leurs stèles funéraires des photographies du (ou de la) disparu(e), reflet d'un ancien culte des ancêtres, et les Tatars d'outre-marais, eux, ont conservé jusqu'à nos jours la coutume qui consiste à déposer sur la tombe des outils du défunt (sa barque, kèmè, par exemple) - toutes choses très peu caractéristiques du rituel islamique.

Il me semble que ce matériau m'autorise à adhérer à l'idée, exprimée dans la bibliographie donnée en cours de texte, que s'est constituée dans les populations türkophones de la plaine de Sibérie occidentale une forme particulière de syncrétisme islam-paganisme, fondée sur la synthèse organique de croyances et de cultes islamiques et préislamiques. Au point qu'il est souvent difficile aujourd'hui, même après de multiples études, de discerner une strate islamique d'une autre non islamique dans les conceptions traditionnelles des populations concernées. Encore fautil rappeler que la catégorie du « paganisme », outre le chamanisme sur lequel nous nous sommes penchés ici, inclut aussi des reliquats de systèmes religieux fort différents les uns des autres (le christianisme, le zoroastrisme...), mais qui tous ont trouvé un reflet direct dans les croyances et les cultes des Tatars et des Qazaqs de Sibérie.

(Traduit du russe par Stéphane A. Dudoignon)

Sibirskoe otdelenie Rossijskoj Akademii Nauk

Omskij filial ob"edinennogo instituta istorii, filosofii i filologii

pr. Mira, 55a

644077 Omsk

30. Cf. T. A. Listova, « Pohoronno-pominal'nye obyčai i obrjady russkih Smolenskoj, Pskovskoj i Kostromskoj oblastej (konec XIX-XX vv.) » (Coutumes et rites funéraires des Russes des régions de Smolensk, Pskov et Kostroma (à la fin du XIX ${ }^{\mathrm{e}}$ siècle et au début du XXe)), in Pohoronno-pominal'nye obyčai (Coutumes funéraires), Moscou, 1993, pp. 58-59; Mify narodov mira (Mythes des peuples du monde), 2e éd., Moscou, 1994, 2, pp. 50-51 ; V. Ja. Propp, Istoričeskie korni vol'šebnoj skazki (Les racines historiques d'un conte merveilleux), Leningrad, 1986, pp. 202, 213-214 ; D. D. Frezer, Fol'klor $v$ Vethom zavete (Le folklore dans l'Ancien Testament), Moscou, 1986, pp. 252-254. 